\title{
PERFORMANCE RATING OF TRANSMUTED NADARAJAH AND HAGHIGHI EXPONENTIAL DISTRIBUTION: AN ANALYTICAL APPROACH
}

\author{
A. A. OGUNDE, A. G. IBRAHEEM and A. T. AUDU \\ Department of Mathematics and Statistics \\ Federal Polytechnic Ado-Ekiti \\ Federal School of Statistics \\ Ibadan \\ Nigeria \\ e-mail: debiz95@yahoo.com \\ ademolaganiyu@gmail.com \\ audu@ymail.com
}

\begin{abstract}
This work investigates the potential usefulness of the transmuted Nadarajah Haghighi exponential distribution for modelling lifetime data. This distribution can be obtained by using the quadratic rank transmutation map scheme. Various structural properties of the transmuted Nadarajah Haghighi exponential model were investigated including moment generating function, order statistics, moment, mean which represent the average life span of a system, variance, estimation of the parameters using maximum likelihood and the potential usefulness of the transmuted Nadarajah Haghighi exponential model was shown by means of Kevlar 373/epoxy data.
\end{abstract}

2010 Mathematics Subject Classification: 65-XX, 70EXX.

Keywords and phrases: quadratic rank transmutation map, moments, Nadarajah Haghighi exponential distribution, maximum likelihood estimation, orders statistics.

Received May 6, 2017; Revised May 17, 2017

(C) 2017 Scientific Advances Publishers 


\section{Introduction}

In this paper, we introduce the transmuted Nadarajah Haghighi exponential distribution which stems from the following idea; Shaw \& Buckley [20] considered composite maps of the following two forms: sample transmutation maps (STMs), $y=G^{-1}(F(x))$ and rank transmutation maps (RTMs), $\nu=G\left(F^{-1}(w)\right)$, where $F$ and $G$ are cumulative distribution functions (CDFs). Gilchrist [9] refers to STMs and RTMs as Qtransformation and P-transformation, respectively. Shaw \& Buckley [21] focused on the RTM, which uses as a tool for the construction of new families of non-Gaussian distributions. They used it to modulate a given base distribution for the purposes of modifying the moments, in particular the skew and kurtosis. An attraction of the approach is that if the CDF and inverse CDF (or quantile function (QF)) are tractable for the base distribution, there is a good chance for the transmuted distribution to be so. According to this approach, a random variable $X$ is said to have a transmuted distribution if its cumulative distribution function (cdf) satisfies the following relationship:

$$
\begin{gathered}
G(x)=(1+\lambda) F(x)-\lambda F(x)^{2}, \quad|\lambda| \leq 1 ; \\
g(x)=f(x)[(1+\lambda)-2 \lambda F(x)],
\end{gathered}
$$

where $F(x)$ is the cdf of the baseline model, $g(x)$ and $f(x)$ are the corresponding probability density functions (pdf) associated with $G(x)$ and $F(x)$, respectively. This paper investigates the statistical properties of the transmuted Nadarajah Haghighi exponential distribution. Aryal and Tsokos [3] studied the transmuted Weibull distribution to analyze two lifetime data sets. Transmuted Lomax distribution (Ashour and Eltehiwy [2]), transmuted exponentiated Gamma distribution (Hussian [10]), transmuted inverse Rayleigh distribution (Ahmad et al. [2]), transmuted Pareto distribution (Merovci and Puka [18]), transmuted 
PERFORMANCE RATING OF TRANSMUTED NADARAJAH ... 139

modified inverse Weibull distribution (Elbatal [6]), transmuted additive Weibull distribution (Elbatal and Aryal [7]), transmuted complementary Weibull geometric distribution (Afify et al. [1]), transmuted inverse exponential (Oguntunde and Adejumo [19]). Khan and King [13, 14] proposed the transmuted modified Weibull and the transmuted generalized inverse Weibull distributions and discussed structural properties with application to reliability data. Khan et al. [12] proposed the transmuted inverse Weibull distribution and discussed various structural properties with application to reliability data. More recently, Khan et al. [15, 16, 17] studied the transmuted generalized exponential, transmuted Weibull, and transmuted generalized Gompertz distributions also, Owoloko et al. [8] examined the performance rating of the transmuted exponential distribution by using QRTM technique which extends the baseline models for modelling lifetime data. Abdul-Moniem and Seham [4, 5] examined the statistical properties of the transmuted Gompertz distribution and also the exponentiated Nadarajah Haghighi exponential distribution.

A random variable $X$ is said to have a Nadarajah and Haghighi exponential distribution (NHED) with shape parameter $\alpha$ and the scale parameter $\beta$, if its probability density function (pdf) is in the form:

$$
f(x ; \alpha, \beta)=\alpha \beta(1+\alpha x)^{\beta-1} e^{1-(1+\alpha x)^{\beta}}, \quad x>0,(\alpha, \beta>0) .
$$

The cumulative distribution function (CDF) and survival function (SF) are:

$$
F(x)=1-e^{1-(1+\alpha x)^{\beta}}, \quad x>0,(\alpha, \beta>0),
$$

and

$$
S(x)=e^{1-(1+\alpha x)^{\beta}}, x>0,(\alpha, \beta>0) .
$$




\section{Transmuted Nadarajah and Haghighi Exponential Distribution}

Using (1) and (4), we can define the CDF of TNHED as follows:

$$
G(x ; \alpha, \beta, \lambda)=\left\{1-e^{1-(1+\alpha x)^{\beta}}\right\}\left\{1+\lambda e^{1-(1+\alpha x)^{\beta}}\right\}, \quad x>0,(\alpha, \beta \text { and } \lambda>0) \text {. }
$$

The pdf of TNHED is given as:

$$
\begin{array}{r}
g(x ; \alpha, \beta, \lambda)=\alpha \beta(1+\alpha x)^{\beta-1} e^{1-(1+\alpha x)^{\beta}}\left[1-\lambda+2 \lambda e^{1-(1+\alpha x)^{\beta}}\right], x>0, \\
(\alpha, \beta \text { and } \lambda>0) .
\end{array}
$$

We can get the pdf for the transmuted exponential (TED), Nadarajah and Haghighi exponential (NHED), and exponential (ED) distributions by taking $\beta=1, \lambda=1$, and $\alpha=\beta=1$, respectively. The survival (reliability) function $S(x)$ and the hazard rate function $h(x)$ for TNHED were obtained as follows:

$$
\begin{aligned}
S(x ; \alpha, \beta, \lambda)=1-\left\{1-e^{1-(1+\alpha x)^{\beta}}\right\}\left\{1+\lambda e^{1-(1+\alpha x)^{\beta}}\right\}, & x>0, \\
& (\alpha, \beta \text { and } \lambda>0),
\end{aligned}
$$

and

$$
\begin{array}{r}
h(x)=\frac{\alpha \beta(1+\alpha x)^{\beta-1} e^{1-(1+\alpha x)^{\beta}}\left[1-\lambda+2 \lambda e^{1-(1+\alpha x)^{\beta}}\right]}{1-\left\{1-e^{1-(1+\alpha x)^{\beta}}\right\}\left\{1+\lambda e^{1-(1+\alpha x)^{\beta}}\right\}}, x>0, \\
(\alpha, \beta \text { and } \lambda>0),
\end{array}
$$

\section{Quantile Function, Median and Mode of the} Transmuted Nadarajah Haghighi Exponential Distribution

The quantile function $x_{q}$ of the TNHED distribution can be obtained as the inverse of Equation (6) 
PERFORMANCE RATING OF TRANSMUTED NADARAJAH ... 141

$$
x_{q}=\frac{1}{\alpha}\left[\left\{1+\ln \left(\frac{(1-\lambda)-\sqrt{(1-\lambda)^{2}+4 \lambda}}{2 \lambda}\right)\right\}^{\frac{1}{\beta}}-1\right] \text {, }
$$

and the median can be obtained as

$$
x_{0.5}=\frac{1}{\alpha}\left[\left\{1+\ln \left(\frac{(1-\lambda)-\sqrt{(1-\lambda)^{2}+4 \lambda}}{2 \lambda}\right)\right\}^{\frac{1}{\beta}}-1\right] .
$$

The lower quartile and upper quartile can also be derived from Equation (11) when $q=0.25$ and $q=0.75$, respectively.

The mode of TNHED is the root of the following equation:

$$
\alpha(\beta-1)\left\{1-(1+\alpha x)^{\beta}\right\}=\alpha \beta(1+\alpha x)^{\beta} e^{1-(1+\alpha x)^{\beta}} .
$$

The graph of cumulative density, probability density, and the hazard functions were plotted below for various values of $\alpha=a, \beta=b$, and $\lambda=b_{1}$.

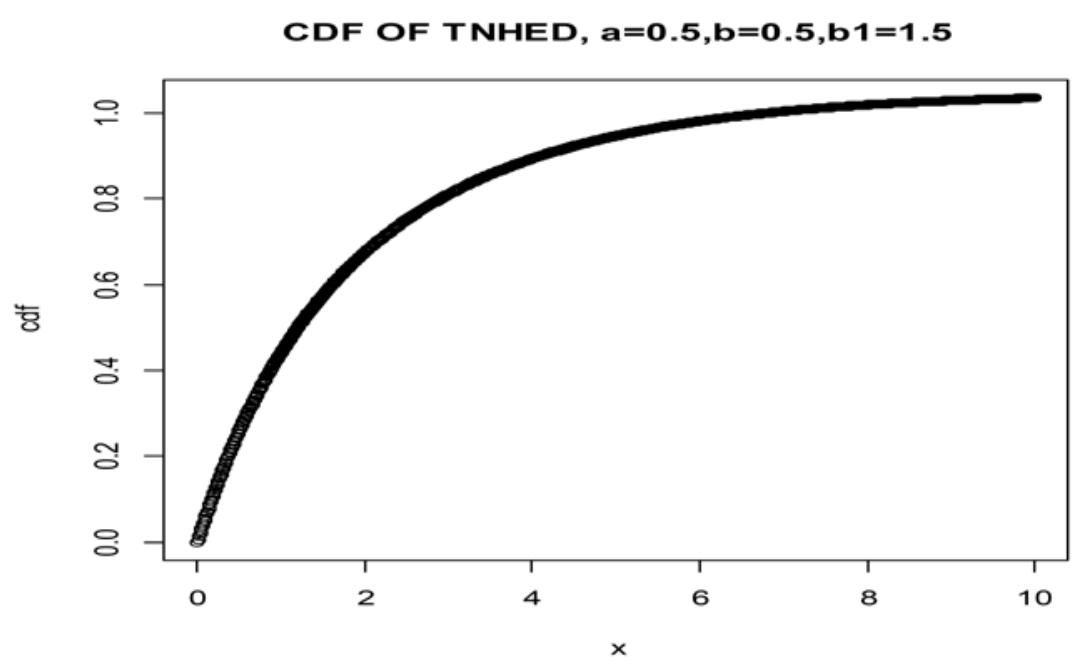

Figure 1. Plot of the cumulative density function for TNHED. 
A. A. OGUNDE et al.
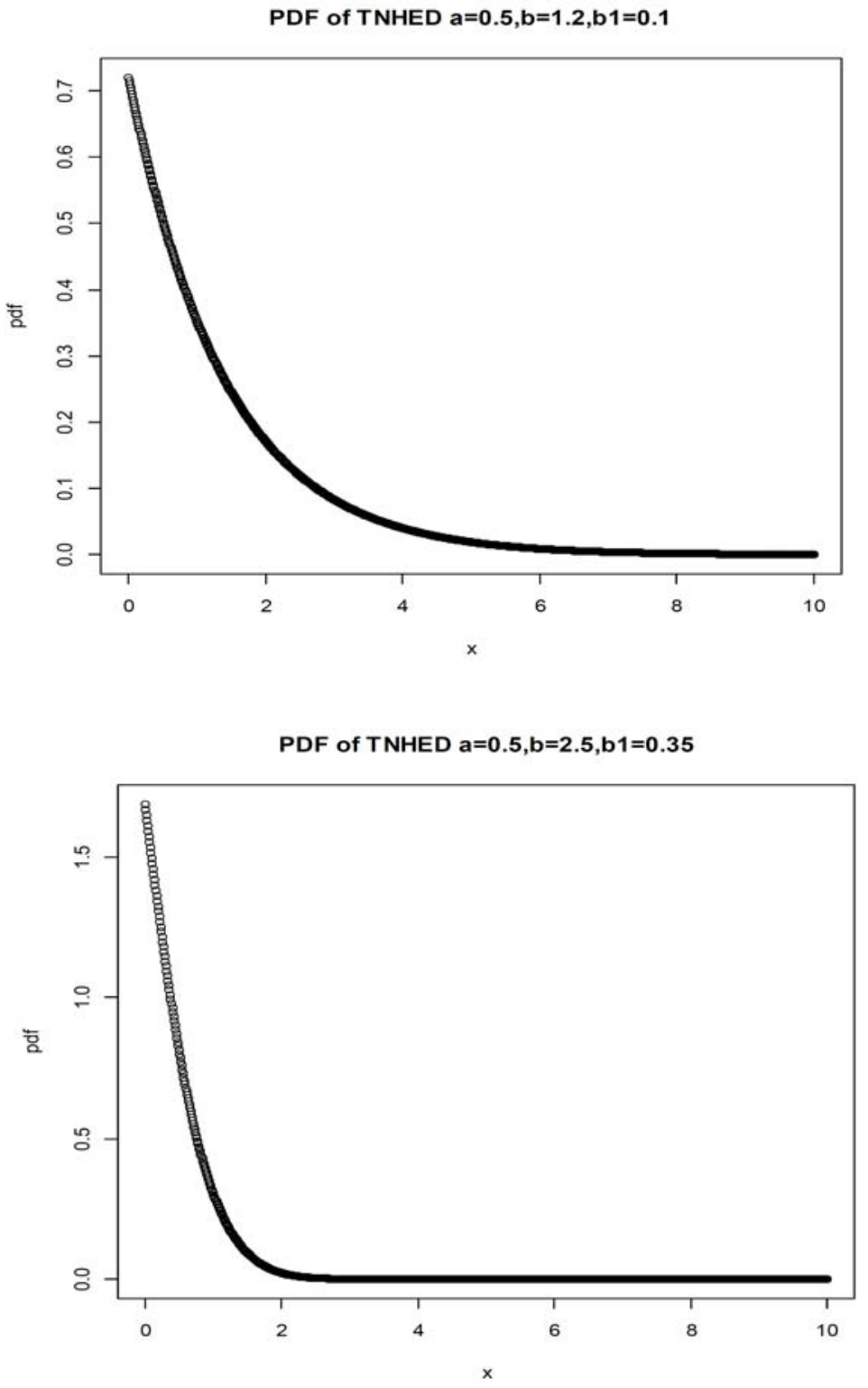

Figure 2. Plot of the density function for TNHED for various values of the parameters. 
PERFORMANCE RATING OF TRANSMUTED NADARAJAH ... 143
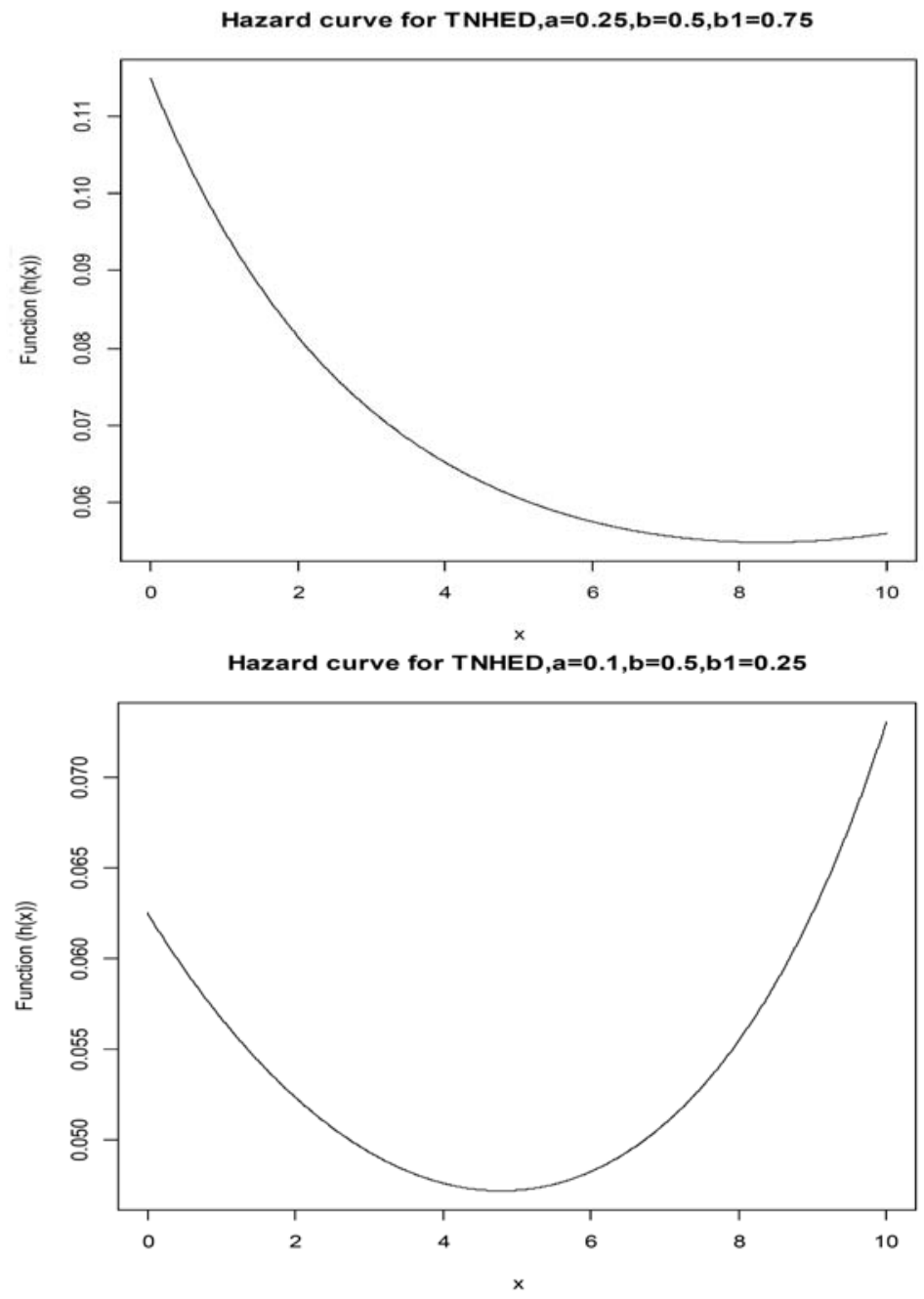

Figure 3. Plot of the hazard function for TNHED for various value of the parameters.

From the diagram above Figure 1 depict that the cdf of TNHED converges to one as $n \rightarrow \infty$ and that shows that the pdf of TNHED is a proper pdf. Figure 2 demonstrates the flexibility of the distribution in modelling lifetime data. Figure 3 gives a clearer picture of the fact that the distribution is reversible both to the right and to the left and it can be used to model lifetime data even those that possess the bathtub-shape failure rate. 


\section{Traditional Moments for TNHED}

Here we obtain $E(1+\alpha x)^{r}$, hence we can obtain the mean and the variance for TNHED as follows:

$$
E(1+\alpha x)^{r}=\alpha \beta \int_{-\infty}^{\infty}(1+\alpha x)^{r}(1+\alpha x)^{\beta-1} e^{1-(1+\alpha x)^{\beta}}\left[1-\lambda+2 \lambda e^{1-(1+\alpha x)^{\beta}}\right] d x
$$

Using the transformation, $1-(1+\alpha x)^{\beta}=-y$, this implies that $1+y=(1+\alpha x)^{\beta}$ and $d x=\frac{1}{\alpha \beta}(1+y)^{\frac{1}{\beta}-1} d y$, then Equation (13) we transform to

$$
E(1+\alpha x)^{r}=\int_{(1+\alpha x)^{\beta}}^{\infty}(1+y)^{\frac{r}{\beta}} e^{-y}\left(1-\lambda+2 \lambda e^{-y}\right) d y
$$

The above equation can be splitted into

$$
\begin{gathered}
I_{1}=\int_{(1+\alpha x)^{\beta}}^{\infty}(1+y)^{\frac{r}{\beta}} e^{-y} d y ; \\
I_{2}=-\lambda \int_{(1+\alpha x)^{\beta}}^{\infty}(1+y)^{\frac{r}{\beta}} e^{-y} d y ; \\
I_{3}=2 \lambda \int_{(1+\alpha x)^{\beta}}^{\infty}(1+y)^{\frac{r}{\beta}} e^{-2 y} d y .
\end{gathered}
$$

Expanding $(1+y)^{\frac{r}{\beta}}$ using the Taylor's series will yield

$$
(1+y)^{\frac{r}{\beta}}=\sum_{l=0}^{\infty}\left(\frac{r}{\beta}\right) y^{l} .
$$


PERFORMANCE RATING OF TRANSMUTED NADARAJAH ... 145

Then expanding Equations (15), (16) and (17) using Equation (18), we obtain

$$
\begin{gathered}
I_{4}=\sum_{l=0}^{\infty}\left(\begin{array}{l}
\frac{r}{\beta} \\
l
\end{array}\right) \int_{(1+\alpha x)^{\beta}}^{\infty} y^{l} e^{-y} d y=\sum_{l=0}^{\infty}\left(\frac{r}{\beta}\right) \Gamma\left(l+1,\left(1+\alpha t_{i}\right)^{\beta}\right) ; \\
I_{5}=-\lambda \sum_{l=0}^{\infty}\left(\frac{r}{\beta}\right) \int_{l}^{\infty} y^{l} e^{-y} d y=-\lambda\left\{\sum_{l=0}^{\infty}\left(\frac{r}{\beta}\right) \Gamma\left(l+1,\left(1+\alpha t_{i}\right)^{\beta}\right)\right\} ; \\
I_{6}=2 \lambda \sum_{l=0}^{\infty}\left(\frac{r}{\beta}\right) \int_{l}^{\infty} y^{l} e^{-2 y} d y=2 \lambda\left\{\sum_{l=0}^{\infty}\left(\frac{r}{\beta}(l) \Gamma\left(l+1,2\left(1+\alpha t_{i}\right)^{\beta}\right)\right\} .\right.
\end{gathered}
$$

Therefore combining Equations (19), (20), and (21), we have

$$
\begin{aligned}
E(1+\alpha x)^{r}= & (1-\lambda)\left\{\sum_{l=0}^{\infty}\left(\frac{r}{\beta}\right) \Gamma\left(l+1,\left(1+\alpha t_{i}\right)^{\beta}\right)\right\} \\
& +2 \lambda\left\{\sum_{l=0}^{\infty}\left(\frac{r}{\beta}\right) \Gamma\left(l+1,2\left(1+\alpha t_{i}\right)^{\beta}\right)\right\} .
\end{aligned}
$$

To obtain the mean of TNHED, we set $r=1$ in Equation (22), then we have

$$
\begin{aligned}
E(1+\alpha x)= & (1-\lambda)\left\{\sum_{l=0}^{\infty}\left(\frac{1}{\beta}\right) \Gamma\left(l+1,\left(1+\alpha t_{i}\right)^{\beta}\right)\right\} \\
& +2 \lambda\left\{\sum_{l=0}^{\infty}\left(\frac{1}{\beta}\right) \Gamma\left(l+1,2\left(1+\alpha t_{i}\right)^{\beta}\right)\right\} .
\end{aligned}
$$

Then we have, 


$$
\begin{aligned}
E(x)= & (1-\lambda)\left\{\sum_{l=0}^{\infty}\left(\begin{array}{l}
\frac{1}{\beta} \\
l
\end{array}\right) \Gamma\left(l+1,\left(1+\alpha t_{i}\right)^{\beta}\right)\right\} \\
& +2 \lambda\left\{\sum_{l=0}^{\infty}\left(\begin{array}{l}
\frac{1}{\beta} \\
l
\end{array}\right) \Gamma\left(l+1,2\left(1+\alpha t_{i}\right)^{\beta}\right)\right\}-\frac{1}{\alpha} .
\end{aligned}
$$

Also setting $r=2$ in Equation (22)

$$
\begin{aligned}
1+2 \alpha E(X)+\alpha^{2} E\left(X^{2}\right)= & (1-\lambda)\left\{\sum_{l=0}^{\infty}\left(\frac{2}{\beta}\right) \Gamma\left(l+1,\left(1+\alpha t_{i}\right)^{\beta}\right)\right\} \\
& +2 \lambda\left\{\sum_{l=0}^{\infty}\left(\frac{2}{\beta}(\Gamma) \Gamma\left(l+1,2\left(1+\alpha t_{i}\right)^{\beta}\right)\right\} .\right.
\end{aligned}
$$

From above, we have

$$
\begin{aligned}
\alpha^{2} E\left(X^{2}\right)= & (1-\lambda)\left\{\sum_{l=0}^{\infty}\left(\frac{2}{\beta}\right) \Gamma\left(l+1,\left(1+\alpha t_{i}\right)^{\beta}\right)\right\} \\
& +2 \lambda\left\{\sum_{l=0}^{\infty}\left(\frac{2}{\beta}\right) \Gamma\left(l+1,2\left(1+\alpha t_{i}\right)^{\beta}\right)\right\}-2 \alpha E(X)-1 .
\end{aligned}
$$

*On simplification we have

$$
\begin{aligned}
& E\left(X^{2}\right)=\frac{1}{\alpha^{2}}\left[(1-\lambda)\left\{\sum_{l=0}^{\infty}\left(\frac{2}{\beta}\right) \Gamma\left(l+1,\left(1+\alpha t_{i}\right)^{\beta}\right)\right\}+2 \lambda\left\{\sum_{l=0}^{\infty}\left(\frac{2}{\beta}\right) \Gamma\left(l+1,2\left(1+\alpha t_{i}\right)^{\beta}\right)\right\}\right. \\
& \left.-(1-\lambda) 2 \alpha\left\{\sum_{l=0}^{\infty}\left(\frac{1}{\beta}\right) \Gamma\left(l+1,\left(1+\alpha t_{i}\right)^{\beta}\right)\right\}+2 \lambda\left\{\sum_{l=0}^{\infty}\left(\frac{1}{\beta}\right) \Gamma\left(l+1,2\left(1+\alpha t_{i}\right)^{\beta}\right)\right\}+1\right] .
\end{aligned}
$$

Then the variance of TNHED is given as 
PERFORMANCE RATING OF TRANSMUTED NADARAJAH ... 147

$$
\begin{aligned}
& \operatorname{Var}(X)=\frac{1}{\alpha^{2}}\left[(1-\lambda)\left\{\sum_{l=0}^{\infty}\left(\frac{2}{\beta}\right) \Gamma\left(l+1,\left(1+\alpha t_{i}\right)^{\beta}\right)\right\}+2 \lambda\left\{\sum_{l=0}^{\infty}\left(\frac{2}{\beta}\right) \Gamma\left(l+1,2\left(1+\alpha t_{i}\right)^{\beta}\right)\right\}\right. \\
& \left.-(1-\lambda) 2 \alpha\left\{\sum_{l=0}^{\infty}\left(\frac{1}{\beta}\right) \Gamma\left(l+1,\left(1+\alpha t_{i}\right)^{\beta}\right)\right\}+2 \lambda\left\{\sum_{l=0}^{\infty}\left(\frac{1}{\beta}\right) \Gamma\left(l+1,2\left(1+\alpha t_{i}\right)^{\beta}\right)\right\}+1\right] \\
& -\left[(1-\lambda)\left\{\sum_{l=0}^{\infty}\left(\frac{1}{\beta}\right) \Gamma\left(l+1,\left(1+\alpha t_{i}\right)^{\beta}\right)\right\}+2 \lambda\left\{\sum_{l=0}^{\infty}\left(\frac{1}{\beta}\right) \Gamma\left(l+1,2\left(1+\alpha t_{i}\right)^{\beta}\right)\right\}-\frac{1}{\alpha}\right]^{2} .
\end{aligned}
$$

\section{Maximum Likelihood Estimators (MLE)}

In this section, we consider maximum likelihood estimators (MLE) of TNHED. Let $x_{1}, x_{2}, \ldots, x_{n}$ be a random sample of size $n$ from TNHED, then the log-likelihood function $l l(\Theta)$ for $\Theta=(\alpha, \beta, \lambda)^{T}$ can be written as

$$
\begin{aligned}
l l(\Theta)=n \log (\alpha) & +n \log (\beta)+(\beta-1) \sum_{i=1}^{n} \log \left(1+\alpha x_{i}\right)+\sum_{i=1}^{n} \log \left[1-\left(1+\alpha x_{i}\right)^{\beta}\right] \\
& +\sum_{n=1}^{\infty} \log \left(1-\lambda+2 \lambda e^{1-\left(1+\alpha x_{i}\right)^{\beta}}\right) .
\end{aligned}
$$

The normal equation become

$$
\begin{aligned}
& \frac{d l}{d \alpha}=\frac{n}{\alpha}+(\beta-1) \sum_{i=1}^{n} \frac{x_{i}}{\left(1+\alpha x_{i}\right)} \\
&+\beta \sum_{i=1}^{n} x_{i}-2 \lambda \beta \sum_{i=1}^{n} \frac{x_{i}\left(1+\alpha x_{i}\right)^{\beta-1} \ln \left(1+\alpha x_{i}\right) e^{1-\left(1+\alpha x_{i}\right)^{\beta}}}{1-\lambda+2 \lambda e^{1-\left(1+\alpha x_{i}\right)^{\beta}}} \\
& \frac{d l}{d \beta}=\frac{n}{\beta}+\sum_{i=1}^{n} \log \left(1+\alpha x_{i}\right)+\sum_{i=1}^{n} x_{i}-2 \lambda \sum_{i=1}^{n} \frac{\left(1+\alpha x_{i}\right)^{\beta} \ln \left(1+\alpha x_{i}\right) e^{1-\left(1+\alpha x_{i}\right)^{\beta}}}{1-\lambda+2 \lambda e^{1-\left(1+\alpha x_{i}\right)^{\beta}}}
\end{aligned}
$$




$$
\frac{d l}{d \lambda}=\sum_{i=1}^{n} \frac{1-2 e^{1-\left(1+\alpha x_{i}\right)^{\beta}}}{1-\lambda+2 \lambda e^{1-\left(1+\alpha x_{i}\right)^{\beta}}} .
$$

The MLE of $\alpha, \beta$, and $\lambda$ can be obtain by solving the Equations (27), (28), and (29), using $\frac{d l l}{d \alpha}=0, \frac{d l l}{d \beta}=0$, and $\frac{d l l}{d \lambda}=0$.

\section{Application of TNHED}

In this section, we use a real data set to show that the TNHED can be a better model than one based on the generalized exponential distribution (GED), transmuted exponential distribution (TED), extended exponential distribution (EED), Nadarajah and Haghighi exponential distribution (NHED), and exponential distribution (ED). We consider a data set of the life of fatigue fracture of Kevlar 373/epoxy that are subject to constant pressure at the $90 \%$ stress level until all had failed, so we have complete data with the exact times of failure. This data are:

$0.0251,0.0886,0.0891,0.2501,0.3113,0.3451,0.4763,0.5650,0.5671$, $0.6566,0.6748,0.6751,0.6753,0.7696,0.8375,0.8391,0.8425,0.8645$, $0.8851,0.9113,0.9120,0.9836,1.0483,1.0596,1.0773,1.1733,1.2570$, $1.2766,1.2985,1.3211,1.3503,1.3551,1.4595,1.4880,1.5728,1.5733$, $1.7083,1.7263,1.7460,1.7630,1.7746,1.8275,1.8375,1.8503,1.8808$, $1.8878,1.8881,1.9316,1.9558,2.0048,2.0408,2.0903,2.1093,2.1330$, $2.2100,2.2460,2.2878,2.3203,2.3470,2.3513,2.4951,2.5260,2.9911$, $3.0256,3.2678,3.4045,3.4846,3.7433,3.7455,3.9143,4.8073,5.4005$, 5.4435, 5.5295, 6.5541, 9.0960 .

In order to compare the distributions, we consider some other criterion like - 2LL, AIC (Akaike information criterion), AICC (Akaike information criterion corrected), and BIC (Bayesian information criterion) for the real data set. The best distribution corresponds to lower - 2LL, AIC, AICC, and BIC, where

$$
A I C=2 P-2 L L, A I C C=A I C+\frac{2 p(p+1)}{n-p-1}, \text { and } B I C=p \log (n)-2 l l,
$$

where $p$ is the number of parameters in the statistical model, $n$ is the 
PERFORMANCE RATING OF TRANSMUTED NADARAJAH ... 149

sample size and $l l$ is the maximized value of the likelihood function for the estimated model. Table 1 shows the exploratory data analysis of the data. Table 2 shows parameter MLE for each one of the fitted distributions. Table 3 shows the values of $-2 \mathrm{LL}$, AIC, AICC, and BIC values.

Table 1. Summary of data on fatigue fracture of Kevlar 373/epoxy at $90 \%$ stress level

\begin{tabular}{|l|l|l|l|l|l|l|l|l|l|}
\hline Min & $\begin{array}{l}\text { Lower } \\
\text { quartile }\end{array}$ & median & $\begin{array}{l}\text { Upper } \\
\text { quartile }\end{array}$ & Mean & Max. & Variance & Skewness & Kurtosis & Range \\
\hline 0.0251 & 0.09048 & 1.7361 & 2.2960 & 1.9590 & 9.0960 & 2.4774 & 1.9406 & 8.1608 & 9.0709 \\
\hline
\end{tabular}

Table 2. Estimated parameters of the TNHED, TED, GED, EED, NHED, and ED

\begin{tabular}{|c|c|c|c|c|}
\hline & \multicolumn{4}{|c|}{ Parametrs estimates } \\
\hline Models & $\alpha$ & $\beta$ & $\lambda$ & $-l l$ \\
\hline TNHED & 0.12371 & -3.5367 & -13.0643 & 39.435 \\
\hline TED & 1.3763 & - & -0.8487 & 121.517 \\
\hline GED & 0.703 & 1.709 & - & 122.244 \\
\hline EED & 0.954 & 6.366 & - & 121.650 \\
\hline NHED & 0.195 & 2.007 & - & 124.738 \\
\hline ED & 0.510 & - & - & 127.114 \\
\hline
\end{tabular}

Table 3. Criteria for comparison

\begin{tabular}{|c|c|c|c|c|}
\hline Models & $-2 \mathrm{LL}$ & AIC & AICC & BIC \\
\hline TNHED & 78.87 & 72.87 & 71.37 & 69.88 \\
\hline TED & 243.034 & 247.033 & 243.198 & 246.796 \\
\hline GED & 244.49 & 244.49 & 248.49 & 103.97 \\
\hline EED & 243.30 & 247.30 & 247.47 & 251.96 \\
\hline NHED & 249.48 & 253.48 & 253.64 & 258.14 \\
\hline ED & 254.23 & 256.23 & 256.28 & 258.56 \\
\hline
\end{tabular}




\section{Conclusion}

It will be observed from the values of the parameters estimates and also the values of the criterion for comparison, the model that contains the minimum information loss which corresponds to minimum $l l$, AIC, AICC, and BIC is considered to be the best model in the class of models considered. An application of the transmuted Nadarajah and Haghighi exponential distribution to real data shows that the new distribution provides a better fit than the generalized exponential distribution, transmuted exponential distribution, exponentiated exponential distribution, Nadarajah Haghighi exponential distribution, and exponential distribution.

\section{References}

[1] A. Z. Afify, Z. M. Nofal and N. S. Butt, Transmuted complementary Weibull geometric distribution, Pak. J. Stat. Operation Res. 10(4) (2014), 435-454.

[2] A. Ahmad, S. P. Ahmad and A. Ahmed, Transmuted inverse Rayleigh distribution: A generalization of then inverse Rayleigh distribution, Math. Theory Model 4(7) (2014), 90-98; S. K. Ashour and M. A. Eltehiwy, Transmuted Lomax distribution, Am. J. Appl. Math. Stat. 1(6) (2013b), 121-127.

[3] G. R. Aryal and C. P. Tsokos, Transmuted Weibull distribution: A generalization of the Weibull probability distribution, European Journal of Pure Applied Mathematics 4 (2011), 89-102.

[4] B. Abdul-Moniem and M. Seham, Exponentiated Nadarajah Haghighi exponential distribution, International Journal of Mathematical Analysis and Applications 2(5) (2015), 68-73.

[5] B. Abdul-Moniem and M. Seham, Transmuted Gompertz distribution, Computational and Applied Mathematics 1(3) (2015), 88-96.

[6] I. Elbatal, Transmuted modified inverse Weibull distribution: A generalization of the modified inverse Weibull probability distribution, Int. J. Math. Arch. 4(8) (2013), 117-129.

[7] I. Elbatal and G. Aryal, On the transmuted additive Weibull distribution, Australian Journal of Statistics 42(2) (2013), 117-132.

[8] Enahoro A. Owoloko, Pelumi E. Oguntunde and Adebowale O. Adejumo, Performance rating of transmuted exponential distribution: An analytical approach, Springer Open Journal 4 (2015), 818. DOI 10.1186/s40064-015-1590-6 
PERFORMANCE RATING OF TRANSMUTED NADARAJAH ... 151

[9] Warren Gilchrist, Statistical Modelling with Quantile Functions, CRC Press Inc., 2000 .

[10] M. A. Hussian, Transmuted exponentiated gamma distribution: A generalization of the exponentiated gamma probability distribution, Appl. Math. Sci. 8(27) (2014), 1297-1310.

[11] M. S. Khan and R. King, Transmuted modified Weibull distribution: A generalization of the modified Weibull probability distribution, European Journal of Pure and Applied Mathematics 6 (2013), 66-88.

[12] M. S. Khan, R. King and I. Hudson, Characteristics of the transmuted inverse Weibull distribution, ANZIAM J. 55 (EMAC2013) (2014), C197-C217.

[13] M. S. Khan and R. King, Transmuted modified Weibull distribution: A generalization of the modified Weibull probability distribution, European Journal of Pure and Applied Mathematics 6(1) (2013a), 66-88.

[14] M. S. Khan and R. King, Transmuted generalized inverse Weibull distribution, Journal of Applied Statistical Sciences 20(3) (2013b), 15-32.

[15] M. S. Khan, R. King and I. Hudson, Transmuted generalized exponential distribution: A generalization of the exponential distribution with applications to survival data, Communications in Statistics: Simulation and Computation, to appear, 2015a.

[16] M. S. Khan, R. King and I. Hudson, Transmuted Weibull distribution Properties and Estimation. Communications in Statistics: Theory and Methods 46(11) (2017), 5394-5418.

[17] M. S. Khan, R. King and I. Hudson, Transmuted Generalized Gompertz distribution: Properties and Estimation, Pakistan Journal of Statistics 32(3), (2016), 161-182

[18] F. Merovci and I. Puka, Transmuted Pareto distribution, Probability Statistical Forum 07 (2014), 1-11.

[19] P. E. Oguntunde and A. O. Adejumo, The transmuted inverse exponential distribution, International Journal Advance Statistics and Probability 3(1) (2015), 1-7.

[20] W. T. Shaw and I. R. Buckley, The alchemy of probability distributions: Beyond Gram-Charlier expansions and a skew kurtotic normal distribution from a rank transmutation map, Research Report, 2007.

[21] W. T. Shaw and I. R. Buckley, The alchemy of probability distributions: Beyond Gram-Charlier expansions, and a skew-kurtotic-normal distribution from a rank transmutation map, 2009. arXiv preprint arXiv:0901.0434. 